\title{
La cultura europea di Foscolo: un bilancio provvisorio
}

La culture européenne de Foscolo : un bilan provisoire

Foscolo's European Culture: A Temporary Synthesis

\section{Enzo Neppi}

\section{OpenEdition}

\section{Journals}

Edizione digitale

URL: http://journals.openedition.org/cei/2281

DOI: 10.4000/cei.2281

ISSN: 2260-779X

\section{Editore}

UGA Éditions/Université Grenoble Alpes

Edizione cartacea

Data di pubblicazione: 30 juin 2015

Paginazione: 7-20

ISBN: $978-2-84310-289-9$

ISSN: 1770-9571

\section{Notizia bibliografica digitale}

Enzo Neppi, «La cultura europea di Foscolo: un bilancio provvisorio», Cahiers d'études italiennes

[Online], 20 | 2015, online dal 01 janvier 2017, consultato il 27 mars 2021. URL: http:// journals.openedition.org/cei/2281 ; DOI: https://doi.org/10.4000/cei.2281 


\title{
LA CULTURA EUROPEA DI FOSCOLO: UN BILANCIO PROVVISORIO
}

\author{
Enzo Neppi \\ Université Grenoble Alpes*
}

Il giovanissimo Foscolo si stabilì nel 1793 a Venezia, in quella che era una delle grandi capitali europee dell'arte e della cultura, dell'editoria e del teatro. Attraverso la frequentazione di Angelo Dalmistro - studioso di letteratura inglese, che aveva tradotto due odi di Gray nel 1792 e curato nel 1794 una raccolta di versioni dall'inglese che includeva versi di Gray, Parnell, Young e Milton - , del cugino Costantino Naranzi, e del salotto di Isabella Teotochi, anch'essa di origine greca, venne presto a contatto con Cesarotti e vari membri della sua cerchia ${ }^{1}$. Conobbe inoltre Aurelio Bertòla - noto autore di una Idea della bella letteratura alemanna (I784) e di un Elogio di Gessner (1789) — e poté leggere, nella traduzione di Mazza, I piaceri dell'immaginazione di Akenside, travasamento poetico di un saggio di Addison. Non può quindi meravigliarci che già nel Piano di Studj² dell'autunno del 1796 risulti amplissima, e quasi preponderante, la cultura europea di Foscolo. Fra i «politici» da studiare Foscolo indica Rousseau e Montesquieu $^{3}$ (che probabilmente diventerà presto per lui un modello

\footnotetext{
* Questo saggio introduttivo è stato scritto da Enzo Neppi, ma vi hanno contribuito con i loro suggerimenti e le loro osservazioni gli altri tre curatori del presente volume. Ringraziamo i membri del comitato scientifico, tutti i colleghi che hanno riletto attentamente i saggi e Marie Conjat per l'editing e la composizione di questo volume.

I. Sulla formazione di Foscolo e la cerchia di Cesarotti, vedi ora C. Chiancone, La scuola di Cesarotti e gli esordi del giovane Foscolo, Pisa, ETS, 2012 e infra Id., L'enseignement de Cesarotti dans la culture européenne du jeune Foscolo : du "Piano di Studj" à l' "Ortis». Per i riscontri fra le letture foscoliane analizzate in questo saggio e le biblioteche, fiorentina e milanese, di Foscolo, vedi infra C. Piola Caselli, Appunti sulla componente europea della biblioteca milanese di Foscolo.

2. EN VI, pp. 3-9, da leggersi tenendo presenti le utili precisazioni di V. Di Benedetto in U. Foscolo, Il sesto tomo dell'To, Torino, Einaudi, I991, pp. 253-259.

3. Sulla cultura francese di Foscolo rimane di grande utilità — per l'esaustività e l'acutezza di alcuni giudizi —, E. Balmas, La biblioteca francese di Ugo Foscolo, in Atti dei Convegni Foscoliani (1978-1979), Roma, Istituto Poligrafico e Zecca dello Stato, I988, vol. II, pp. 215-233. 
alternativo al Contrat social) ${ }^{4}$, fra i «metafisici» l'antimetafisico Lockes, ma accanto a lui anche il cartesiano e malebranchiano Yves-Marie André, autore di un trattato sul bello, e fra i logici il leibniziano Wolff. Tuttavia, per il giovane Foscolo, «chiave universale di ogni filosofia» è Bacone di Verulamio ${ }^{6}$.

Fra gli storici Foscolo accosta Raynal ${ }^{7}$ a Tacito, ma fra i moderni cita un Midleton che potrebbe essere George Lyttelton, amico di Pope, Fielding e Thomson, e autore di una History of the Revolutions of England (I768). Ricorda inoltre Voltaire (pur biasimato per scarsa meditazione) e Jean-François de La Harpe, per i suoi lavori enciclopedici di storia e di geografia. Fra i poeti il Piano di Studj menziona abbastanza scontatamente Ossian, Gray, Thomson, Young, le Lettere di Abelardo e Eloisa di Pope, lo svizzero Haller ${ }^{8}$ e Klopstock ${ }^{9}$; fra i satirici (accanto a Parini) Boileau, per il suo Lutrin; fra i romanzieri Fénelon (per il Telemaco), Swift (per La novella della botte), Fielding ${ }^{\mathrm{IO}}$ e Baculard d'Arnaud, ma poi soprattutto La nouvelle Héloïse, dalla quale Foscolo risale a Richardson ${ }^{\mathrm{II}}$ e Goethe. Nell'Ortis 1798 saranno fruiti anche episodi di Wieland e di Bernardin de Saint-Pierre, eliminato quest' ultimo nelle successive edizioni, dove Sade fa invece un'apparizione pregnante benché fugace.

Fra i teorici e gli studiosi dell'arte Foscolo ricorda Marmontel, Mengs e Winckelmann, a prova del suo interesse, che sempre rimarrà vivo, per $\mathrm{i}$ teorici del neoclassicismo. Una o due giovanili produzioni poetiche, oggi perdute, dimostrano quanto contasse per lui la figura di Robespierre ${ }^{\mathrm{I} 2}$, che

4. Poco più tardi, agli inizi del 1798 (EN VI, p. 57) compare anche il nome di Pufendorf, ricordato però come storico, per la sua storia della Svezia in latino (I679). Ma è sicuramente precoce in Foscolo l'attenzione al dibattito europeo sul diritto naturale e l'origine dello stato, inseparabile dalla riflessione su Machiavelli.

5. Sull'importanza di Locke per Foscolo, vedi, in questo volume, M. Palumbo, Foscolo e Locke.

6. Bacone sarà di nuovo ricordato nella Chioma di Berenice per la sua «operetta d'oro De sapientia veterum» (EN VI, p. 366), nell'edizione delle opere di Montecuccoli per il suo lavoro sulla "catena delle scienze», allusione probabile al De augmentis (EN VI, p. 620), nei frammenti su Machiavelli e nelle Lettere dall'Inghilterra, come indicheremo più avanti.

7. La ricorrente condanna in Foscolo della tratta degli schiavi e delle atrocità patite dai negri è certo legata alla lettura della Histoire des deux Indes di Raynal.

8. Di Haller (scienziato e poeta amato fra gli altri da Kant) Pagani Cesa aveva tradotto, nel I782, Die Ewigkeit (1736), meditazione escatologica sul nulla e l'eternità.

9. Quest'ultimo sarà ricordato nella Chioma di Berenice per il volo dell'angelo Eloa nel canto ottavo del Messia (EN VI, p. 345).

Io. Foscolo ricorda nel Piano di Studj, e poi anche nell'Ortis 1798, "Amalia» (EN IV, p. I36). Si tratta quasi sicuramente del romanzo di Fielding, di cui Foscolo doveva aver letto l'adattamento molto libero di M.-J. Riccoboni: Amélie, roman de Fielding traduit de l'anglois par Mme Riccoboni (I743). Fielding sarà menzionato di nuovo negli anni inglesi, per le dissertazioni che aprono i diversi libri del Tom Jones (EN V, p. 262).

II. Foscolo alluderà più tardi a Clarissa in una lettera ad Antonietta Fagnani Arese, accusata poi anche di essere «il Lovelace femminile» (Ep. I, pp. 367, 4II).

I2. EN VI, pp. 7, 9, 4I; EN II, p. 343. 
sempre rimarrà (con Napoleone) al centro della sua riflessione, all'inizio, almeno in certi momenti, come modello, poi come spauracchio. Foscolo menziona anche Cervantes ${ }^{13}$ e, fra i tragici, Voltaire e Shakespeare ${ }^{14}$, ma ignora, e continuerà a lungo a ignorare, la letteratura non italiana del Medio Evo e del Quattrocento europei ${ }^{15}$.

La seconda parte dell'Ortis 1798 (che probabilmente risale al I7961797) conferma alcuni di questi nomi, cui si potrà aggiungere il Voltaire dell'Alzire e il poeta sepolcrale tedesco Zachariae, ma tutto l'Ortis, nelle sue diverse stesure, fino alla Notizia bibliografica zurighese, scritta in parte come risposta alle riflessioni di Goethe sul proprio romanzo in Dichtung und Wahrheit, riposa su due massicci pilastri: il Werther e la Nouvelle Héloüse ${ }^{16}$. Rousseau è senz'altro l'autore che più ha segnato il giovane Foscolo — fino a un certo momento anche come modello di vita — nei diversi registri del suo pensiero. Per difendersene e liberarsene, sia come romanziere che come pensatore politico, Foscolo avrà bisogno di dosi massicce di Locke, Sterne ${ }^{17}$

13. Come noto, Cervantes è per Foscolo una delle stelle più luminose di una nebulosa scettica e/o comica di cui fanno parte anche Rabelais, Montaigne, Molière, Bayle, Sterne, Swift e altri. Riguardo a Bayle (che conta anche come erudito, ed è biasimato da Foscolo come anti-machiavelliano), Balmas ricorda in particolare una lettera alla contessa d'Albany in cui Foscolo lo giudica "l'intelletto più eroico che abbia creato mai la natura» per la sua capacità, dopo avere trovato «in tutte le cose discordia, [...] errore e notte perpetua", di «tenere aperti gli occhi in quel Caos» (Ep. VI, p. I54).

I4. Foscolo evoca, nella sua prima lettera a Cesarotti (30 ottobre I795), l'Atrée et Thyeste (1707) di Crébillon padre, e i Pélopides di Voltaire (I772) come fonti di un suo primo Tieste a noi non pervenuto (Ep. I, p. I9). Corneille e Racine non figurano nel Piano di Studj né ci risulta che siano stati spesso citati in seguito. L'Ortis I802 conterrà invece una trasparente allusione a Lady Macbeth. Nella Chioma di Berenice Shakespeare sarà ricordato come chi insegna al volgo inglese «gli annali patrii», ma anche per il «soprannaturale mirabile e orrendo degli incantesimi», che "nasce nei tempi barbari» (EN VI, pp. 308, 393). Nella Notizia intorno a Didimo Chierico, Shakespeare è paragonato a una «selva incendiata che faceva bel vedere di notte, e mandava fumo noioso di giorno" (EN V, p. I80), a prova di un certo persistente pregiudizio 'voltairiano' nei suoi confronti. Una pagina destinata al Gazzettino del bel mondo, oltre a rimproverare a Shakespeare di avere «adulato gentilmente la pazzia» della regina Elisabetta, ricorderà come egli «propendesse per la monarchia assoluta, ch'ei paragona [in Troylus and Cressida] al Sole regolatore de' moti di tutte le stelle». A chi, come Monti, volesse trarre da questa similitudine una giustificazione di Napoleone, Foscolo replica argutamente, con una battuta in cui si riassume tutto il suo pensiero politico, e tutta la sua filosofia dell'armonia dissonante: «il sole che riscalda troppo - egli scrive - e che non lascia tornare le stelle e la luna a risplendere su la terra, la ridurrebbe tutta quanta peggio de' deserti dell'Africa» (EN V, p. 4IO). Ma negli anni inglesi i rimandi a Shakespeare diventano sempre più frequenti e nella corrispondenza accadrà più volte a Foscolo di paragonarsi al malinconico Hamlet, antesignano, con Yorick, di Didimo Chierico e Jacopo Ortis. A Foscolo si deve, a quanto pare, anche un breve articolo anonimo On Hamlet del «New Monthly Magazine» (I82I) costruito come commento a una celebre analisi della tragedia nel Wilhelm Meister di Goethe (EN X, pp. 583-589).

I5. Molto più tardi, in una lettera a Lord Holland, Foscolo menzionerà con riverenza «il vecchio Chaucer» (il cui nome ritorna con una certa frequenza negli anni inglesi), ma ammettendo di non intenderne facilmente la lingua arcaica (Ep. VII, p. 273).

I6. Sull'intertestualità europea dell'Ortis nelle sue diverse stesure, e sull'influsso cruciale di Goethe e Rousseau, vedi E. Neppi, Il dialogo dei tre massimi sistemi: Le "Ultime lettere di Jacopo Ortis» fra il «Werther» e la "Nuova Eloisa», Napoli, Liguori, 20I4 (con una lunga sezione iniziale di storia della critica).

17. Su Sterne e la cultura inglese di Foscolo (con attenzione particolare a Locke e Hume) vedi da ultimo S. Parmegiani, Ugo Foscolo and English Culture, London, MHRA, $201 \mathrm{I}$ e infra Id., Da Sterne alla critica dei 
(già presenza importante nella prima parte dell'Ortis $\mathrm{I} 798$, e naturalmente nel Sesto tomo dell'Io), Gray, Machiavelli (pure letto anche lui attraverso Rousseau) e altri.

Difficile dire se già in parte a Venezia nel 1797 o solo più tardi, a Milano e in Francia, Foscolo abbia conosciuto l'opera dei continuatori francesi del sensismo di Locke e di Hume e di altri rappresentanti del tardo illuminismo francese (Condillac, Helvétius, Barthélemy, Volney) ${ }^{\mathrm{r}}$, e quella di critici della religione su base erudita come Dupuis e Bailly (il che non gli impedisce di citare anche il più ortodosso Antoine Pluche), sempre però controbilaciati nella sua mente dalla riflessione vichiana sul mito, la religione e la fantasia. La Chioma di Berenice mostra inoltre dimestichezza con filologi di scuola tedesca e inglese, che continueranno a essere coltivati anche in seguito (Bentley, Heyne ${ }^{19}$, Creech, Döring, Valckenaer, Voss, Vulpius e Withof), con un umanista del XVI secolo come Marc-Antoine Muret, con il quarto libro dell'Émile di Rousseau, con Gibbon e con un altro storico inglese, con cui Foscolo dialogherà anche negli anni seguenti: William Roscoe (I753-183I) autore di un'importante Life of Lorenzo de Medici (1796), cui seguirà dieci anni dopo The Life and Pontificate of Leo the Tenth (I805).

I Sepolcri racchiudono un dialogo aspramente polemico con i maggiori rappresentanti della poesia sepolcrale europea, da Parnell a Pindemonte (ma nel carme si possono anche identificare citazioni quasi letterali da Louis de Fontanes, Delille e Joseph-François Michaud). Il carme è inoltre indizio di un'appassionata partecipazione al dibattito sulle inumazioni suscitato dalla soppressione dei riti di sepoltura cristiani nella Francia rivoluzionaria, dibattito in cui fra gli altri intervennero Roederer (su posizioni radicalmente naturaliste e materialiste), Bernardin de SaintPierre e vari difensori del rito cattolico ${ }^{20}$.

Un po' più tardi, l'edizione delle opere di Montecuccoli (I808) sarà un'occasione per ricordare - a proposito degli eloquenti elogi a Turenne di Saint-Évremond, Bossuet e Fléchier — che in quegli anni «la preponderanza

romanzi inglesi nell'Epistolario di Foscolo. Una diversa angolatura teorica sulla ricezione di Sterne in Foscolo è qui proposta da A. Moioli nel suo saggio La trace de Sterne dans les romans autobiographiques de Foscolo.

I8. Come aveva già suggerito Mario Fubini, Francesco Lomonaco è stato probabilmente un mediatore importante fra Foscolo e le correnti sensiste e materialiste del tardo illuminismo francese. Cfr. C. Piola Caselli, I "Discorsi letterari e flosofici» di Francesco Lomonaco nelle lezioni pavesi di Ugo Foscolo, in M. Tortora e S. Tinterri (a cura di), L'ottimismo della volontà. Studi per Giovanni Falaschi, Perugia, Morlacchi, 20II, pp. 85-IoI.

19. Su Heyne, vedi in questo volume C. Del Vento, Una nuova lettera di Ugo Foscolo.

20. Sull'intertestualità europea dei Sepolcri vedi L. Sozzi, I «Sepolcri» e le discussioni francesi sulle tombe, "GSLI», LXXXIV, 1967, pp. 567-588, e i tre saggi di Bertazzoli, Neppi e Sozzi in G. Barbarisi e W. Spaggiari (a cura di), "Dei Sepolcri» di Ugo Foscolo, Milano, Cisalpino, 2006. 
di Luigi XIV [...] disanimava gli scrittori dalla verità». A queste scritture agiografiche Foscolo contrappone la biografia in francese di Andrew Michael Ramsay (I686-I743), «libro utilissimo all'arte storica e militare ed alla scienza morale e politica», e il Siècle de Louis XIV di Voltaire, che non esita a denunciare «le stragi e gl'incendi del Palatinato». Foscolo accenna inoltre alle ricerche di storia militare svolte nelle scuole di Justus Lipsius e Johannes Meursius (I579-I639), cui rimprovera l'incapacità di «risalire alle ragioni universali delle vittorie greche e romane», cita Puységur (I6561743), autore di un Art de la guerre pubblicato postumo dal figlio nel I748, Jean-Charles de Folard (I669-I752) per i suoi studi sulla guerra e l'arte militare in Polibio, Joly de Maiseroy (I719-1780), autore di un Traité des stratagèmes permis à la guerre (1765), Lancelot Turpin de Crissé (1716-1793) noto per il suo Commentaire sur les "Mémoires de Montecuculi» (sic; 1769) e per un Essai sur l'art de la guerre, infine Bousmard (I749-1807) per il suo Essai général de fortification et d'attaque et défense des places (I797-I802).

Nello stesso volume, Foscolo si rifa più volte alla Défense du système de guerre moderne e al Journal du voyage en Allemagne di Guibert (I744I790), steso quest'ultimo con "coscienza di storico, per sacrificare alla verità», mentre il medesimo aveva scritto un Éloge du roi de Prusse (1787) "per destare in Francia l'emulazione marziale», come già era stato indicato nel I803 dalla «Décade littéraire». Ricorda le Vies des hommes illustres [et grands capitaines] étrangers di Brantôme (I540-I6I4), e il satirista tedesco Johannes Balthazar Schupp (I6IO-I66I) autore di una Dissertatio [...] de opinione (1640), in cui Machiavelli è difeso dalle ingiuste calunnie dell'opinione. Cita il "proemio» all'Encyclopédie di Diderot e D'Alembert ${ }^{21}$, il Montesquieu della Grandeur et décadence des Romains (cap. XVI) e dell'Esprit des lois (III, 5), ma soprattutto difende «l'assioma di Hobbes e i corollari di Machiavelli e di Montesquieu», da cui si ricavano "i veri e soli diritti della guerra che Ugo Grozio desunse dai fatti», facendo svanire il mondo ideale di «Platone, Cicerone, Rousseau». Foscolo conclude lodando - con l'abituale senso dell'antitesi, pregnante espressione della dualità umana - il Montecuccoli che insegnava nelle sue opere «a devastare in guerra le campagne», ma "puniva la ferocia militare che affligge stolidamente l'agricoltore», al fine di «insegnare ad avere pietà de' popoli nelle fatali necessità della guerra ${ }^{22}$.

2I. Sono frequenti, anche se spesso fugaci, le allusioni di Foscolo a D'Alembert. Ci limitiamo qui a segnalare un articolo inglese, in cui il padre dell'Encyclopédie è ricordato per avere giustificato la critica letteraria, necessaria come gli "occhiali che aiutano chi è corto di vista" (EN XI/2, p. 569).

22. EN VI, pp. 617, 597, 599, 678, 607, 616n. I, 620, 665, 615, 618. 
Parallelamente, nell'Orazione pavese Foscolo prosegue il dialogo con il Bacone del De augmentis e del De sapientia (letti sicuramente in latino), polemizza con l'egualitarismo di Rousseau e di Cartesio, e utilizza non solo i già ricordati Barthélemy, Bailly, Dupuis e Condillac, quest'ultimo studiato quasi sicuramente attraverso Lomonaco, ma anche la Historia critica philosophiae di Brucker e il De origine obeliscorum di Georg Zoega; affronta argomenti che erano discussi in quegli anni in giornali da lui frequentati, come la "Décade philosophique» e la «Décade égyptienne»; dialoga polemicamente con le filosofie del progresso che erano state elaborate da Mandeville, Vico, Turgot e Kant, benché sia difficile stabilire, a parte Vico, in che misura li avesse letti; mostra una conoscenza di La Rochefoucauld e di La Bruyère, e di quelli che chiama i «Pensieri cristiani» di Pascal, accostato sul tema della noia a Helvétius in una lettera a Giambattista Giovio del 29 settembre i808. La traduzione di un passo del Pascal di Contre l'indifférence des athées, a sua volta derivato dalla Apologie de Raymond Sebond, è riprodotta nell'orazione Sull'origine e i limiti della giustizia, e poi di nuovo, in una delle ultime pagine dell'Ortis I8I6, ma senza mai indicarne l'autore ${ }^{23}$. Nel 1808 Foscolo mostra interesse anche per la Geografia fisica di Kant e forse per l'Essay on the History of Civil Society (1767) di Ferguson, che poteva leggere in traduzione francese o italiana.

Come noto, negli anni successivi, fra Milano e Firenze, e di nuovo a Milano, fino alla fuga in Svizzera, Foscolo è variamente occupato, oltre che da esose polemiche letterarie italiane, dall'Ajace, dalla Ricciarda, da riflessioni su Machiavelli, dalla traduzione del Sentimental Journey di Sterne ${ }^{24}$, e dalle Grazie (in cui l'episodio del cigno votivo mostra familiarità con l'opera di Buffon). Non può quindi sorprenderci che siano meno numerose le figure della cultura europea che vediamo affiorare per la prima volta in questo periodo ${ }^{25}$. Il saggio Degli effetti della fame e della disperazione sull'uomo, del I8II, mostra tuttavia con quanta attenzione Foscolo abbia studiato il Voyage dans la Haute Pensylvanie et dans l'État de New-York (I8oI) di St John de Crèvecour. Nelle Considerazioni sui "Pensieri

23. EN VII, pp. I82-I83; EN IV, pp. 455-456. Vedi E. Neppi, La traduzione come suicidio simbolico: Un frammento pascaliano dell'Ortis, "Franco-Italica», I996, n I0, pp. 69-8I.

24. Nelle cui note, e nella cui successiva Notizia (oltre ad autori che abbiamo già ricordato, come Raynal, per il suo elogio di «Eliza», Delille, Hume, Montaigne, Rabelais, Cervantes, ecc.) sono menzionati Roger Ascham (I515-1568), citato attraverso Baretti (Gavazzeni), il drammaturgo tedesco August von Kotzebue, il dizionario di Samuel Johnson, Smollett, Hume, il tragediografo John Home (I722-I808), lo storico Charles James Fox (I749-I806), Les égarements du coeur et de l'esprit di Crébillon fils, il Catone di Addison, Lavater e un saggio di Vicesimus Knox (I752-I82I) su Sterne.

25. Sulle risonanze europee delle Grazie, fra l'altro in poeti ignoti a Foscolo, o che non riuscirono a destare la sua attenzione, vedi, in questo volume, il saggio di C. Lombardi, Atlantide e altri "pays chimériques». 
intorno al "Principe"》 di Angelo Ridolfi, egli passa in rassegna - ma senza averle studiate direttamente - le opere di numerosi machiavelliani e antimachiavelliani europei, da Hubert Languet (I5I8-I58I), che attribuisce all'influenza di Machiavelli la strage degli Ugonotti, a Conring, Harrington e Federico II di Prussia. Ma la lettura di Angelo Ridolfi e di Roscoe finirà col convincerlo a rinunciare alla lettura 'repubblicana' del Principe ${ }^{26}$. A questo periodo risalirebbe anche l'interesse di Foscolo per le tesi storiografiche di Sismondi sul Medio Evo italiano.

$\grave{E}$ inoltre degno di nota che emerga in questo periodo il nome di Schiller, annoverato in una conversazione con von Orelli fra "gli otto o nove maggiori poeti di tutti i tempi». Di lui Foscolo legge la «Storia de' trent'anni», ma anche l'Ode alla gioia e soprattutto alcune tragedie di cui loda «le maschie ed originali bellezze» ${ }^{27}$. Peraltro, la composizione delle Grazie, l'amicizia di Cicognara e i quasi quotidiani incontri fiorentini con la contessa d'Albany non potevano non favorire un intenso rapporto intellettuale con François-Xavier Fabre, rapporto che culmina nell'abbozzo di una lettera proemiale sulla poesia d'Omero, il «maggiore de' poeti che abbiano saputo dipingere». Come ricorda Barbarisi nella sua introduzione, le principali fonti delle note erudite di Foscolo ai suoi Esperimenti di traduzione dell'Iliade sono da ricercare in Cesarotti e nei commentatori da lui menzionati: francesi come Anne e André Dacier, inglesi come il Pope, traduttori e commentatori in latino (Cunich, Heyne, Clarke), scoliasti raccolti da Villoison nella sua edizione del manoscritto Venetus A dell'Iliade (Venezia, I788). Dallo stesso lavoro di traduzione di Omero nasceranno i saggi omerici concepiti in Inghilterra, l'incompiuto articolo sulla Storia del testo d'Omero e la History of the Aeolic Digamma, in cui la riflessione di Foscolo è arricchita dalla conoscenza degli studiosi britannici: Bentley, Blackwell, Dawes, Samuel Clarke, i più recenti Marsh e Burgess, e in particolare Richard Payne Knight, frequentato amichevolmente da Foscolo sin dal principio del $\mathrm{I} 8 \mathrm{I} 7^{28}$.

Più importante (e in stretto legame con la lettera a Fabre) è l'approfondimento in questo momento, se non la scoperta, del Laocoonte di Lessing ${ }^{29}$. Significativa inoltre l'amicizia di William Stewart Rose,

26. Su tutta questa questione, vedi EN VIII, pp. 19-39 e soprattutto A. Ridolfi, U. Foscolo, Scritti sul "Principe» di Niccolò Machiavelli, a cura di P. Carta, C. Del Vento e X. Tabet, Rovereto, Nicolodi, 2004, pp. 3I-49, I29-I72.

27. Ep. IV, pp. II3n., II4, I43.

28. EN III/I, pp. 2I5-243 (2I8 per la citazione), XXXIII n. I; EN XII, p. XXXVIII.

29. Su cui vedi, infra, E. Parrini Cantini, Foscolo e l'estetica di Lessing e C. Del Vento, Una nuova lettera di Ugo Foscolo (con appunti anche su Madame de Staël, Heyne e la sua edizione delle Georgiche). 
membro del comitato editoriale della "Quarterly Review», i cui consigli certo contribuirono alla successiva decisione di Foscolo di lasciare Zurigo per l'Inghilterra. Foscolo discorre con lui della tragedia di Otway, Venice Preserved, che ricorderà ancora nei frammenti dell'incompiuto articolo sulla Costituzione aristocratica di Venezia, denunciandone la scarsa affidabilità storica ${ }^{30}$.

Degna di nota, durante il soggiorno svizzero, l'amicizia del zurighese Jacob Heinrich Meister (I744-I826) che, dopo essere stato costretto all'esilio da un proprio scritto del 1769 (De l'origine des principes religieux), era stato prima segretario di Melchior Grimm, e poi continuatore per molti anni della sua Correspondance littéraire. Meister è ricordato nella Notizia bibliografica per un' «operetta sull'immortalità dell'anima», Euthanasie (di cui il poeta aveva iniziato la traduzione) e a lui Foscolo penserà più tardi come dedicatario di una delle Lettere dall'Inghilterra, quella sull'esilio. Proprio in una lettera a Meister da Francoforte, in data 30 agosto I8I6, Foscolo racconta sgomento l'incontro con Friedrich Schlegel, che gli fa l'elogio della Santa Inquisizione, del papa e del «medio evo, la più illuminata di tutte le epoche da Adamo in poi» ${ }^{3 \mathrm{I}}$.

In Svizzera Foscolo fu in relazioni d'amicizia anche con Johann Heinrich Füssli (1745-1832), studioso di storia e di belle arti, succeduto al Bodmer nell'insegnamento della storia patria al Collegium Carolinum di Zurigo, socio di quella libreria Orell e Füssli presso cui Foscolo pubblicherà sia i Vestigi della storia del sonetto italiano che l'Hypercalypseos liber e l'Ortis zurighese. Corrispose inoltre con il pastore di origine italiana Johann Kaspar von Orelli (I787-I849), che acquisterà più tardi solidissima fama di filologo classico.

Con l'arrivo a Londra, ai primi di settembre del I8I6, la passione per la cultura inglese, che risaliva all'adolescenza, diventa esperienza vissuta, immersione nel territorio e quotidiano commercio umano. Ma Foscolo, in Inghilterra, spiegò soprattutto agli inglesi la cultura italiana, e nonostante stretti legami con i più appassionati cultori delle civiltà antiche e moderne del sud dell'Europa, numerosi anche a causa della 'moda italiana' allora imperante, rimase estraneo sia all'orientamento spiritualista di alcuni grandi autori romantici (Wordsworth, Coleridge e Southey), sia agli sviluppi più recenti del genere romanzesco, che s'incarnavano per lui nei romanzi storici di Walter Scott e in un romanzo di argomento italiano

30. Ep. V, p. 254; EN XII, p. 658.

3I. EN IV, pp. 527-528; EN V, p. 252; Ep. VI, p. 559. 
come Valperga di Mary Shelley Wollstonecraft ${ }^{32}$. Foscolo sentì più affine a sé Byron, di cui cita e ammira, oltre alla condizione di esule, il Childe Harold e il Marin Faliero. Spesso rimproverò inoltre agli autori inglesi di non conoscere abbastanza l'Italia o di trascurare le ricerche erudite di studiosi come Muratori, Zeno, Tiraboschi, Maffei, Bianchini (che invece Newton - scrive orgogliosamente Foscolo - aveva riconosciuto come «uno de" grandi astronomi» del suo tempo) ${ }^{33}$. Ma nel corso degli anni inglesi Foscolo acquistò una dimestichezza con la lingua e con la letteratura inglese che fino ad allora gli era mancata. Consapevole inoltre di rivolgersi a un pubblico i cui riferimenti culturali sono diversi da quelli del lettore italiano, cita ora autori che raramente facevano capolino nelle sue opere precedenti $\mathrm{i}^{34}$.

Come noto, le Lettere scritte dall'Inghilterra ${ }^{35}$ furono uno dei primi progetti letterari concepiti da Foscolo dopo il suo arrivo nell'isola, e lo occuparono per circa un anno, fra la primavera del I8I7 e il marzo dell'anno seguente. Solo uno studio approfondito delle carte labroniche permetterà di determinare l'estensione delle letture di questo periodo. Ma già le pagine fin qui pubblicate ci forniscono utili indicazioni. È per esempio degno di nota che nell'introduzione "Al lettore» Foscolo menzioni un "ginevrino" (Rousseau) e un «inglese» (Hobbes), come i due "grandi maestri che hanno esaminato l'uomo in stato di natura». Dei due il secondo, «d'anima più vigorosa; ma sgomentata naturalmente da terrori panici», considerava l'uomo «un tigreconiglio [che] merita d'essere disprezzato e in catene». Alcuni prìncipi che seguirono i suoi precetti «morirono profughi», un altro, «un grande monarca» che, per lo stesso motivo, «incatenò [...] la razza umana europea», si trova ora «sotto la zona torrida», paga cioè le conseguenze dei propri atti nell'isola di Sant'Elena ${ }^{36}$.

Sia nelle Lettere che nel successivo saggio sulla Italian Periodical Literature del '24 (in cui molte pagine sono dedicate a Baretti) Foscolo cita più volte Samuel Johnson, lodandolo per la magnificenza delle sentenze - senza la quale perderebbero molto del loro valore - per le ricchezze

32. Vedi, infra, il saggio già citato di Sandra Parmegiani. Riflessioni analoghe, con riferimento all'atteggiamento di Foscolo nei confronti della tragedia romantica, sono svolte in questo volume da Borsa e Del Vento nel saggio su Foscolo, Manzoni e la cerchia di Byron. Sulla ricezione europea del teatro di Foscolo vedi inoltre il saggio di P. Ranzini, Foscolo auteur de tragédies.

33. EN XI/2, p. 3 IO.

34. Sulla dimensione greca (e politica) del Foscolo inglese, vedi invece, infra, il saggio di Angelo Colombo su Foscolo, Mustoxidi e gli esuli pargioti.

35. Su cui vedi la scheda introduttiva di Elena Lombardi in U. Foscolo, Opere II, Prose e saggi, Torino, Einaudi-Gallimard, 1995, pp. 935-943.

36. EN V, pp. 240-24I. 
della sua mente, cresciute spontaneamente, senza bisogno di studi ordinati, per le tante verità applicabili alle letterature di ogni tempo e paese. A Johnson Foscolo riconosce anche il merito di avere forgiato un nuovo codice critico, che poi fu introdotto da Baretti in Italia, ma gli rimprovera pregiudizi, sofismi e la sonora rotondità dello stile, spiegabile forse con l'intenzione di fare dimenticare le proprie umili origini. Foscolo non gli perdona però il giudizio severo sull'Aminta del Tasso, subito peraltro anche da Pindaro e Gray, né condivide il rimprovero, fatto a Milton, di essere stato nella vita privata «un tiranno», mentre pubblicamente si proclama «difensore indomabile della libertà». Egli loda poi in particolare The Life of Samuel Johnson di Boswell, di cui ci dice che sarebbe quello da lui prescelto, se fra tutti i libri di biografia moderna gliene si lasciasse uno solo. Foscolo ammira il fatto che da tanti minimi detti e accidenti Boswell abbia saputo spremere più sostanza di storia letteraria che non da tutto il bel esprit degli elogi di Fontenelle, o dalle lunghe liste di date del Tiraboschi ${ }^{37}$.

In un altro appunto in francese, destinato anch'esso a confluire nelle Lettere dall'Inghilterra, Foscolo s'interroga sul breve manoscritto autobiografico di quattro pagine, My own Life, steso da Hume nell'aprile I776, quattro mesi prima di morire. Lo colpisce la calma profonda con cui il filosofo "parcourt presque d'un seul instant toute la toile de sa vie, l'espèce d'indifférence dont il trace son passage du berceau au tombeau comme s'il lui devait être égal de vivre ou de mourir ou de n'être jamais né», e si chiede se lo stile di quelle pagine denoti un autentico «equilibrio delle passioni» o non nasca piuttosto da una certa dose d'affettazione; ma conclude che l'anima di David Hume non doveva aver bisogno di molti sforzi per apparire così distaccata. Hume, che già era stato, sul piano del carattere, l'anti-Rousseau, ci si presenta qui come l'anti-Foscolo, come un modello di serenità forse agognata ma irraggiungibile ${ }^{38}$.

Nello stesso periodo Foscolo si mette a studiare Corinne ou l'Italie di Madame de Staël, che lo interessa anche come resoconto del viaggio in Italia di uno straniero. Una lettera a Miss Pigou, che gli ha regalato il romanzo, ce lo mostra intento a riempire uno scartafaccio di appunti col libro aperto sul tavolino. Per lui Madame de Staël, nonostante il «bellissimo ingegno", è vittima della Metafisica, che l'ha spinta a sciorinarci

37. EN V, pp. 425-426; EN XI/2, pp. I92-193, 319, 344, 348-351, 354.

38. EN V, pp. $275-276$. 
"teorie incomprensibili con neologismo tedesco ${ }^{39}$, entusiasmo ginevrino» e «amabilità femminile»; «a fondare avvenimenti storici sopra la favola»; a credere che basti "galoppare seco ${ }^{40}$ in carrozza" per "penetrare in un voltar d'occhi negli usi, nelle opinioni, nella letteratura, e nelle viscere delle nazioni». Foscolo coglie insomma l'occasione per mettere in guardia contro ciò che ci accade quando la Metafisica si mischia «alla poesia, alla rettorica e all'arte d'amore», facendo «avvampare la mente d'idee» e ravvolgendo «di nebbia luminosissima le umane azioni e passioni, in guisa che nessuno possa discernere ciò che è da ciò che non è, e nemmeno da ciò che non può essere mai». Era più saggio, secondo Foscolo, «il grande Bacone», (da lui definito vent'anni prima, come si è visto, «chiave universale di ogni filosofia»), "metafisico d'altra fatta», il quale ben sapeva che gli uomini non amano la verità nuda e cruda, che sempre preferiscono mescolarla con vane opinioni e lusinghiere speranze: «A mixture of a lie doth ever add pleasure» o, nella delicata traduzione di Foscolo, «un po' di tinta di bugia aggiunge garbo alla verità» ${ }^{4 \mathrm{I}}$.

Nel I8I8 Foscolo avvia anche quegli studi danteschi che culmineranno nella pubblicazione di un'edizione della Commedia nel i825. Nella prefazione ai due volumi dell'Edizione Nazionale che raccolgono gli Studi su Dante, Giovanni Da Pozzo si sofferma sulle letture di cui si avvalse Foscolo nelle sue ricerche. Foscolo trovò in Warburton, curatore di un'importante edizione di Shakespeare (1747), i precetti che si sforza di seguire anche lui: "correggere gli errori testuali, mettere in luce le peculiarità del linguaggio, chiarire le allusioni, spiegare bellezze e imperfezioni del sentimento». Non meno importante fu la lettura di due vaste sintesi storiche: la recente View of the State of Europe during the Middle Ages (I8I8), primo grande lavoro di Henry Hallam (I777-I859), e A View of the Progress of Society in Europe (1769) dell'illuminista scozzese William Robertson, noto da tempo a Foscolo per la sua History of the Reign of the Emperor Charles $V$; e in più una delle più autorevoli cronache latine del XIII secolo, la Historia anglorum, di Matthew Paris. Per i Saggi sul Petrarca Foscolo si avvalse invece soprattutto della preziosa collaborazione di Lady Dacre (I768-I854), che gli fornì le traduzioni in inglese dei versi di Petrarca, e delle ricerche erudite dell'abbé de Sade, Mémoires pour la vie de François Pétrarque (Amsterdam, I764-I767). In modo simile, l'ultimo saggio pubblicato in vita da Foscolo,

39. Qui il riferimento è a De l'Allemagne. "L'entusiasmo ginevrino» allude invece all'influenza di Rousseau e al comune retroterra protestante.

40. Il pronome riflessivo si riferisce alla Metafisica: è lei, personificata, che scarrozza per l'Italia la romanziera. 4I. Ep. VII, p. 222; EN V, pp. 254, 364-365, 375. 
la History of the Democratic Constitution of Venice saccheggia ampiamente la monumentale Histoire de la République de Venise di Daru (I8I9), pur ribadendo contro di lui nello stesso tempo le origini democratiche di Venezia, e dissentendo dalla tesi del suo fatale declino.

Il I822 è l'anno in cui Foscolo collaborò al catalogo descrittivo dei marmi di Woburn Abbey ${ }^{42}$. Come noto, questa era la dimora familiare dei duchi di Bedford, e racchiudeva dal I8I9, come in un prestigioso museo, il gruppo delle Grazie scolpito da Canova per John Russell, sesto duca di Bedford. Curatore del volume e autore dell'introduzione al catalogo, traduttore, inoltre, delle pagine in prosa di Foscolo, fu Philip Hunt (I772I838), archivista del duca. Ma Foscolo collaborò di sicuro alla descrizione del gruppo statuario con alcune pagine che spiegano l'origine del culto delle Grazie, compose i successivi 90 versi («Mentre opravan le Dee») e scrisse la Dissertation on an Ancient Hymn to the Graces, in cui sono interpolati 7 frammenti poetici, fra i quali figurano nuovamente i 90 versi appena indicati.

Spendiamo infine qualche parola sulla cultura inglese dispiegata da Foscolo negli articoli, di argomento prevalentemente italiano, che sono raccolti nel volume XI/2 dell'Edizione Nazionale. Narrative and Romantic Poems of the Italians, pubblicato nell'aprile I8I9 nella "Quarterly Review», recensisce la traduzione di William Stewart Rose degli Animali parlanti di Casti e un'imitazione del Morgante Maggiore di Pulci ad opera di John Hookham Frere (1769-I846). A parte questi due autori, vi è ricordato in primo luogo Dryden, lodato per l'«admirable versification» del poemetto satirico The Hind and the Panther ma criticato per avere «denaturalized the character of the Apologue». Dello stesso Dryden Foscolo ricorda A Song for St. Cecilia's Day (I687), certo a lui noto da tempo dal momento che Angelo Mazza, poeta frequentato da Foscolo sin dall'adolescenza, si era ispirato a Dryden e aveva dedicato alla santa due odi sull'armonia. Foscolo unisce tuttavia Dryden a Monti nel rimprovero di avere reso la poesia «subservient to their private interests, at the expense of truth and honour», trasparente allusione al fatto che Dryden, dopo avere parteggiato per Cromwell, si era convertito al cattolicesimo al momento della restaurazione di Carlo II.

Milton è ricordato da Foscolo come un profondo conoscitore di Pulci, e il Paradise Lost è lodato come opera che è stata all'origine della rivoluzione inglese per le sue idee religiose, ma è di interesse per tutta l'umanità; è inoltre accostato a Dante e Tasso in quanto non concepì la letteratura

42. Di cui si raccomanda la recente riedizione: Outline Engravings and Descriptions of the Woburn Abbey Marbles (M.DCCC.XXII). Le Grazie a Woburn Abbey, a cura di A. Bruni, Firenze, Polistampa, 2012, 2 voll. 
come mero divertimento ma scrisse "with heartfelt warmth and dignity on subjects [...] sublime and beautiful in themselves and important to the world»; ed è menzionato per le riflessioni di poetica svolte nella prefazione a Samson Agonistes, paragonate da Foscolo a quelle analoghe di Corneille, Racine, e Voltaire.

Foscolo menziona alcuni altri poeti: William Cowper (I73I-I80o), autore di un poemetto, The Task (1785), da lui descritto come quanto di più simile al Giorno si fosse fatto in Inghilterra in quegli anni; Samuel Rogers (I763-1855), cui Foscolo dedica l'Ortis londinese del I8I7, e reso a suo tempo famoso dai Pleasures of Memory (1792), che chiudevano la stagione preromantica inglese; George Crabbe (I754-I832) poeta disincantato dei poveri (The Village, I783; The Borough, I8Io) ${ }^{43}$.

Più significativa è forse la pagina in cui Foscolo difende Muratori dalle obiezioni di Gibbon alla sua erudizione libresca. «Le date de' fatti - sostiene Foscolo - sono nella storia ciò che le note musicali sono nell'armonia d'un'orchestra». Gibbon, Roscoe e Sismondi non avrebbero mai potuto scrivere i loro vasti lavori di sintesi senza le ricerche erudite di Muratori, Tiraboschi e Apostolo Zeno: Gibbon corre la terra col «carro del sole», «e guarda il suo predecessore col trionfo del giovane David davanti al vasto gigante», ma avrebbe mai potuto percorrere una così «magnifica porzione della storia del genere umano» se Muratori non lo avesse preceduto con i suoi «lenti e cauti» annali? ${ }^{44}$

Nei Classical Tours (1824) Foscolo confronta i viaggi in Italia di Addison (Remarks on Several Parts of Italy, 1705), Eustace (A Classical Tour through Italy, I8I5) e Forsyth (Remarks on Antiquities, I8I4, che il Dictionary of National Biography definiva ancora, alla fine del XIX secolo, come «one of the best books on Italy in our language»).

Il giudizio di Foscolo sulle tre opere è perentorio. Di Addison dice che è il "primo e fra tutti elegante», di Eustace, che è il "più voluminoso e fantastico», di Forsyth, che è «il più accurato» e il più «originale». Considerazioni morali e politiche non sono forse del tutto estranee a questo giudizio. Chiudono infatti il saggio le parole di Forsyth sul Colosseo: «Moralists may tell us that the truly brave are never cruel; but this monument says "no". Here sat the conquerors of the world cooly to enjoy the tortures and death of men who had never offended them» ${ }^{45}$.

43. EN XI/2, pp. 33, I7I, I75, 409, 4I9, 466; EN V, p. 404.

44. EN XI/2, p. 317.

45. EN XI/2, pp. 286-297, 267. 
Questa commossa denuncia in inglese dei conquistatori che godono a torturare le proprie vittime ci sembra chiudere degnamente questo panorama, che in realtà si è limitato a preparare il discorso svolto nei saggi che seguono e a offrire qualche spunto per ricerche future. Di alcuni di questi saggi, approfonditi scandagli su ciò che qui è stato solo accennato, abbiamo già detto. Ma tutta una parte di questo volume è dedicata alla variegata ricezione e diffusione di Foscolo in diversi paesi europei — Francia, Polonia e Grecia - , e all'immagine della sua opera e del suo personaggio in alcune grandi figure dell' 'emigrazione' italiana reale: Da Ponte, Santorre di Santarosa, Mazzini; o ideale: Gadda.

Questa raccolta di saggi è dedicata alla memoria di due grandi amici e straordinari studiosi di Foscolo: Umberto Carpi e Franco Longoni. 